\title{
Por uma geografia da música: um panorama mundial e vinte anos de pesquisas no Brasil
}

\author{
Lucas Manassi Panitz
}

Mestre em Geografia. Doutorando no Programa de Pós-graduação em Geografia. Universidade Federal do Rio Grande do Sul et Université Bordeaux 3. E-mail: lucaspanitz@gmail.com

Recebido em 04/2012. Aceito para publicação em 12/2012.

Versão online publicada em 01/02/2013 (http://seer.ufrgs.br/paraonde)

\begin{abstract}
Resumo - A geografia da música, apesar de quase um século de existência oficial, só recentemente têm tido a devida atenção dos geógrafos interessados no estudo da cultura e das manifestações artísticas em sua dimensão espacial. A quantidade de materiais disponíveis em formato digital atualmente permite um bom reconhecimento deste campo de estudo em geografia, e indica Estados Unidos, Inglaterra e França como centros de discussão avançada, além do Brasil como potencial âmbito ibero-americano, por sua considerável produção acadêmica. Neste sentido, é apresentado um panorama mundial nos estudos de Geografia e música, e um balanço dos vinte anos de pesquisa da Geografia brasileira.

Palavras-chave: Geografia. Música. Geografia da Música. Geografia Brasileira.
\end{abstract}

\section{Introdução}

A música há algum tempo vem despertando um crescente interesse na Geografia, embora ainda incomparável a outras ciências humanas como a Antropologia, a Sociologia e os Estudos Culturais. Contudo, há de ressaltar que a gênese do interesse das ciências humanas pela música remete, justamente, à gênese da Geografia moderna, da Etnologia e da Arqueologia. A Geografia da música, apesar de quase um século de existência oficial, só recentemente têm tido a devida atenção dos geógrafos interessados no estudo da cultura e das manifestações artísticas em sua dimensão espacial. A quantidade de materiais disponíveis em formato digital atualmente permite um bom reconhecimento deste campo de estudo em geografia, e indica Estados Unidos, Inglaterra e França como centros de discussão avançada. No âmbito ibero-americano verifica-se sua pouca difusão, com exceção do Brasil, onde se encontra um considerável número de teses e dissertações produzidas nos últimos vinte anos, além de artigos e traduções de artigos seminais na temática. Neste sentido, será apresentado um panorama global destes estudos, e uma apresentação destes vinte anos de pesquisa no Brasil, marcados pela diversidade de abordagens e pela valorização da cultura brasileira.

\section{As raízes da discussão}

Ao contrário do que se possa imaginar, quan- do tratamos de manifestações culturais e espaço geográfico, o interesse geográfico pela música não aparece no giro cultural dos anos 1980, quando decorridas as reorientações teóricas nas ciências sociais, em especial nos países anglo-saxões. As primeiras considerações que ligam a Geografia moderna à expressão musical podem ser atribuídas à Friedrich Ratzel e seu discípulo Leo Frobenius, etnólogo e arqueólogo africanista. Como observa Reynoso (2006), Ratzel influenciou decididamente a Escola Histórico Cultural alemã e austría$\mathrm{ca}$, sendo Frobenius o principal pesquisador que levou adiante as teorias do geógrafo alemão. Atento aos indícios materiais da cultura, Ratzel observou similaridades entre os arcos da África Ocidental e da Melanésia, suas características morfológicas, bem como as formas das flechas usadas junto com o arco. Frobenius levou a pesquisa adiante e relacionou similaridades entre os tambores e outros instrumentos musicais, que o levou a desenvolver a noção de Círculos Culturais (Kulturkreis) junto aos etnologistas austríacos Fritz Graebner e Wilhelm Schimidt, inspirados em Ratzel. Partindo dessa noção, a partir do estudo da distribuição espacial de instrumentos musicais, entre outros procedimentos, Frobenius estabeleceu regionalizações na África que remetem aos ciclos de difusão de etnias, propondo, por exemplo, a seguinte divisão: Negra, MelanésioNegra, Indo-Negra e Semítico-Negra (MERRIAM, 1983). Estabelecendo áreas culturais a partir de uma espacialidade dos instrumentos musicais na África, Leo Frobenius pode ser considerado o primeiro sis- 
tematizador do estudo entre espaço geográfico e música, que irá influenciar toda uma geração de etnólogos e musicologistas. Dessa forma, na busca de uma gênese do interesse da Geografia moderna pela música, até o presente momento encontramos em Ratzel o princípio inspirador dessa discussão, bem como em Frobenius o desenvolvimento teórico e empírico da mesma.

É bom lembrar que a noção de Kulturkreis postulava que certo número de ciclos culturais se desenvolvia em todo o mundo, em distintos lugares, em distintas épocas históricas e se difundiam no espaço, dando origem a novas culturas; essas proposições basearam-se em princípios difusionistas, que influenciaram diretamente a Antropologia e a Geografia Cultural norte-americanas, a partir da Universidade de Berkeley. Carl Sauer, filho de imigrantes germânicos radicados nos Estados Unidos, foi o fundador da Geografia Cultural americana, também chamada de Escola de Berkeley. Sauer foi profundamente influenciado pelas teorias difusionistas de Ratzel, utilizando-se da noção de Área Cultural de seu colega, o antropólogo Alfred Kroeber (este, discípulo do prussiano Franz Boas, a quem se deve a criação da noção de Área Cultural, e responsável por levar aos Estados Unidos uma versão renovada da Kulturkreis). Carl Sauer, profundo leitor da obra de Ratzel, herda o interesse pelos estudos de difusão e, sobretudo pela noção de Área Cultural, de Boas e Kroeber, amplamente relacionada com os postulados de Ratzel. Vendo assim, não se estranha que o interesse geográfico pela música tenha se dado justamente com os discípulos de Sauer, e que, como veremos adiante, sejam os Estados Unidos e o Canadá aqueles que desenvolvem até hoje boa parte das publicações na área.

Porém, tal interesse não foi exclusividade dos países germânicos e dos Estados Unidos. Nas duas primeiras décadas do século XX, na França, são desenvolvidas reflexões acerca de uma "Geografia musical" como disciplina própria. Nesse sentido o etnólogo, arqueólogo e geógrafo francês Georges de Gironcourt propõe esse novo campo de estudos para a geografia nos Annales de Géographie da Associação Francesa de Geografia (GIRONCOURT, 1927), tendo realizado diversos estudos na Tunísia, Java e Camboja. Com a intenção de estabelecer um novo campo de estudo na Geografia, o autor afirma que "pode-se admitir que o repertório de sons eles mesmos e de suas associações em combinações musicais foram até agora negligenciados pelos geógrafos." (ibidem, p. 292). Segundo o autor, a Geografia musical deveria se debruçar sobre as formas musicais através do espaço e do tempo, permitindo analisar a fixação e a mobilidade de sociedades e civilizações. A simples introdução, por exemplo, de certo tipo de chocalho em uma banda de jazz norte-americana, pode dar informações importantes sobre a origem étnica e geográfica de determinados grupos, mas também de instrumentos e formas musicais que vão se transformando e se adaptando a cada sociedade em que são inseridos. É assim que, em trabalho posterior, Gironcourt (1939) expõe alguns dos resultados pessoais coletados ao longo de doze anos dedicados ao tema, desde seu artigo seminal advogando pela nova disciplina geográfica. No referido texto, o autor afirma através de diversos estudos realizados pelo mesmo, que é possível recompor a mobilidade de populações e suas origens através das formas musicais, pois estas formas permanecem no tempo e no espaço ao longo da história humana ou se modificam levando algumas características pretéritas para outros lugares: ou seja, há um caráter de fixidade e um caráter de mobilidade dos grupos humanos os quais podem ser estudados através da música.

A principal diferença entre Frobenius e Gironcourt é que enquanto o primeiro reconstituía períodos históricos e pré-históricos através da cultura material, ao segundo também interessavam as formas não materializadas como os ritmos, o canto e as danças tradicionais. Gironcourt permanece desconhecido até hoje, tendo sido o novo corpo editorial da revista La Géographie responsável por resgatar a importância desses artigos seminais quando da organização de uma edição especial sobre geografia e música. Dessa forma, considerase que desde as últimas publicações de Gironcourt, ao final da década de 1930, a geografia francesa permaneceu distante do tema, até que grupos de geógrafos retomaram seu interesse, e de forma mais sistemática, dentro de unidades de pesquisas do CNRS - Centre National de la Recherche Scientifique.

\section{As geografias musicais anglófonas}

Nash \& Carney (1996), dois dos principais precursores do tema na América do Norte, fazem um retrospecto das últimas três décadas de trabalhos na área, no mundo anglófono. No esforço de atribuir uma origem à geografia da musica, e sem citar à Frobenius e Gironcourt, os autores afirmam que

A pré-história da geografia da música foi dominada por etnomusicologias e folcloristas, os quais focaram não só nos tipos e localizações de instrumentos musicais, 
mas também em regiões musicais. Curt Sachs, e seu Geist un Werden der Musikinstrumente (Significado e Desenvolvimento de Instrumentos Musicais) publicado em 1929 [...] e outros profundamente interessados nos aspectos espaciais de suas pesquisas etnomu-sicológicas (idem, p.70).

Os autores ainda avaliam que desde a década de 1960 até 1996, mais de quarenta artigos haviam sido publicados em revistas internacionais e nacionais e quase o mesmo número de papers sobre o tema foram apresentados em encontros de geografia e ciências humanas. Eles ainda atribuem a conferencia "The Place of Music" organizado pelo Instituto de Geógrafos Britânicos e as sessões especiais de geografia da música na Associação de Geógrafos Americanos, ambos realizados na primeira metade da década de 1990, como notáveis indícios da credibilidade da disciplina, considerada pelos mesmos com um subcampo da geografia cultural; essa credibilidade, segundo os autores, também foi corroborada pelas citações dos pesquisadores da área em atlas, enciclopédias, bibliografias, livrostextos de geografia humana e livros acadêmicos. Influenciados pela Geografia cultural da Escola de Berkeley, os geógrafos anglófonos (sobretudo estadunidenses e cana-denses) focaram-se boa parte do tempo em descrição das representações espaciais nas canções, nas análises locacionais e de difusão de ritmos, instrumentos e práticas musicais, e na regionalização destes.

Contudo, a partir da a conferência The Place of Music em 1993, organizado pelo Instituto de Geógrafos Britânicos, surge uma renovação dos estudos, em direção a abordagens mais críticas. De fato, a conferência aparece em um momento de franca renovação da geografia cultural nos países de língua inglesa, levado adiante em grande medida por pesquisadores britânicos, e já passado mais de uma década desde o artigo "0 supra-orgânico na geografia cultural americana” de Duncan (1980), onde o autor faz uma crítica à geografia cultural saueriana, e que é considerado um dos momentos mais importantes da virada cultural na geografia anglo-saxã. A obra The Place of Music reúne artigos de pesquisadores esta-dunidenses, canadenses e, sobretudo britânicos, do campo da geografia, da etnomusicologia, da história, dos estudos culturais, entre outros. Logo em sua introdução, seus organizadores tratam de expor a perspectiva da coletânea. Se opondo ao tratamento dado pela music geography de influência saueriana, como George Carney, Peter Nash, entre outros, os autores afirmam:

o trabalho geográfico sobre música teve até pouco recentemente uma tendência de restringir-se ao mapeamento de difusão de estilos musicais, ou analisar o imagético geográfico nas letras de canções, trabalhando com um restrito deliberado senso de geografia, oferecendo o ângulo de um geógrafo fincado ao chão, ao invés de se perguntar o quanto uma abordagem geográfica pode reconfigurar o próprio chão que pisa. Ao contrário, nós proce-demos com uma compreensão que, ao injetar geografia na música, poderá produzir um efeito análogo a que David Harvey advoga na relação com a teoria social: "Ao inserir conceitos de espaço em qualquer teoria social, se produz um efeito de borrar/confundir as proposições centrais daquela teoria". The Place of Music apresenta espaço e lugar não como simples locais onde a música é fabricada, ou de onde ele é difundida/ ao invés disso, diferentes espacialidades são sugeridas como for-madoras do som. [...] Considerar o lugar da música não é reduzi-la a sua localização, estabelecer um ponto exato no espaço, mas permitir uma abordagem rica em estéticas, culturas, economias e geografias políticas da linguagem musical (LEYSHON et al 1998, p.4).

Seguindo esta corrente de estudos é que ganhou notoriedade a geógrafa singapurense Lily Kong e sua tese de doutorado sobre música, políticas culturais, identidade e globalização em Singapura. Kong foi uma das primeiras que se tem notícia que trouxe para o debate teórico da geografia - já nesta fase de renovação - a formulação de uma análise geográfica da música popular. Kong (1995) afirma que, como interesse geográfico, a música não foi explorada largamente e os estudos publicados até então mantiveram uma distância das questões teóricas e metodológicas da geografia cultural renovada. Em artigos posteriores, Kong (1996, 1997) explora a construção das identidades locais e dos processos de trans-culturação em Singapura através da música popular, expondo sua tese no sentido de que "apesar de um mundo com tendências globalizantes, as fronteiras não estão inteiramente apagadas. De fato [...] onde o cruzamento de forças globais é mais forte, a afirmação do local é maior, conco-mitantemente" (1997, p.10).

Analisando atualmente alguns dos principais periódicos de geografia do mundo anglófono, é possível visualizar um fértil panorama dos estudos da música em geografia, com bom número de geógrafos trabalhando nessa perspectiva - em muitos dos casos relacionados com a agenda que Kong propusera - tais como Anderson, Morton \& Revill (2005), Connel \& Gibson (2004), Finn (2009), Florida \& Jackson (2009), Gibson (1998, 2009), Hogan (2007), Hudson (2006), Jazeel (2005), Kearney (2008), Kingsburry (2008), Kruse (2004), Revill 
(2000, 2005), Saldanha (2005), entre outros. Em geral nesses trabalhos, e seguindo a tendência da geografia cultural anglo-saxônica, o binômio "space and place" continua sendo o mais utilizado para os estudos que envolvem geografia e música. Notase igualmente forte influência dos estudos culturais na formulação teórica dos trabalhos.

\section{A perspectiva francesa e o foco no território}

Se os artigos pioneiros do tema são atribuídos a Gironcourt, os próximos textos encontrados que retomam a discussão - já com propostas renovadas - datam da década de 1990. Nesse sentido, vemos em Lévy $(1994,1999)$ uma retomada com interessantes observações teóricas e filosóficas ao tema. Em seu artigo, Lévy usa o panorama musical erudito europeu para contextualizar seus distintos desenvolvimentos, seus condicionantes políticos e culturais e as difusões de inovação musical por seu território. Analisando os processos sociaispolíticos-econômicos que contribuíram para uma cena de vanguarda musical na Viena dos inícios do séc. XX, caracterizada pela atonalidade, Levy afirma que a questão da identidade de um território e de suas manifestações artísticas tem a ver com o cruzamento de distintas espacialidades - interfaces complexas entre redes, territórios e regiões. Romagnan (2009), por sua vez, advoga pela música como um novo campo de estudos para a geografia. 0 autor não remonta o interesse até à época de Gironcourt, mas sim aos artigos seminais de Lévy e Lechaume (1997), à abordagem cultural de Paul Claval e Jean-Pierre Augustin, e defende o diálogo da ciência geográfica com a sociologia da música e a etnomusicologia. Romagnan introduz alguns aspectos importantes da música como uma atividade de grande importância cultural e social, explica a contribuição de sociólogos, antropólogos e etnomusicólogos, e insere a idéia da atividade musical como um geo-indicador do território ao abordar temas como política cultural, música e espaço público, sistemas de produção dessa atividade, uso dos lugares de práticas musicais e seus significados, entre outros temas.

Outros autores como Calenge (2002, sobre geografia econômica da cultura e redes da indústria cultural), Lamantia (2002, tratando dos efeitos territorializantes das músicas de ambiente, como supermercados e lojas), Goré (2004, sobre a construção territorial e identitária a partir da música e dança tradicionais), reforçam a abordagem territorial da geografia francesa atual nos estudos sobre a música. Na sistematização desse campo de estudos, temos Guiu (2006) e Raibaud (2008) como os principais articuladores de organização de periódicos, colóquios e coletâneas de artigos. Raibaud afirma que "a música aparece como uma realidade cognitiva possível para compreender o espaço das sociedades, inclusive como um princípio de organização territorial" (idem, p.2). Para este autor, a música "borra os mapas: sua fluidez se adapta à organização em redes, conexões, ramificações (Amselle, 2001), a música se multiplica com as tecnologias da informação e da comunicação" (ibidem).

Dessa forma, verifica-se que muito embora o interesse da geografia francesa pela música seja recente, os trabalhos contemporâneos mostram-se bem organizados e têm no território, em suas diversas abordagens, a sua principal categoria de análise geográfica.

\section{Geografia e música no Brasil: vinte anos de pes- quisas e pluralidade de interesses}

Ao completar vinte anos de pesquisa sobre música na Geografia brasileira, iniciado com a dissertação de Mello (1991), é possível fazer um breve balanço. Embora não sejam numerosas, as pesquisas realizadas demonstram uma heterogeneidade de abordagens, usando a música para trabalhos de caráter humanista e abordagens culturais renovadas, enfoques da geografia social, ou como ferramenta para o ensino. Em termos conceituais, também, encontramos diversidade nas abordagens, ora focando-se na paisagem, ora no espaço geográfico, ora na região, ora no território. Serão expostos nesta seção os trabalhos contidos no banco de teses e dissertações da CAPES, e artigos em periódicos e coletâneas de Geografia.

Considera-se que João Baptista Ferreira de Mello tenha sido o precursor do tema na geografia brasileira, com sua dissertação defendida na UFRJ em 1991. É a partir de autores como David Seamons, David Ley, Antoine Bailly e Douglas Pocock, que Mello se inspira para interpretar a cidade do Rio de Janeiro sob a ótica de seus compositores, no período de 1928 à 1991, trabalhando na perspectiva da canção como uma "literatura musicada". Mas será somente após uma década do trabalho de Mello que a música passará a ser um interesse constante e crescente na Geografia brasileira. A partir da década de 2000, e com maior projeção após a segunda metade da mesma, se observa uma intensificação da música como objeto de estudo geográfico. Nesse sentido serão mostradas possíveis linhas de abordagem e de interesse temático.

Desde o trabalho percursor de Mello (1991), o interesse pela música surge atrelado à 
geografia humanística. Em sua tese (MELLO, 2000), o autor trata dos mundos vividos da cantora Marlene, apoiado nas filosofias do significado (fenomenologia, existencialismo, hermenêutica) em direção a uma geografia do indivíduo. Importante ressaltar que o mesmo passa posteriormente a orientar trabalhos sobre música (Quadro 1), seguindo os interesses que inaugurara em sua dissertação: a música popular brasileira (em especial o samba) e o Rio de Janeiro. Assim, trabalhos como os de Guimarães (2007), Santos (2009), Pizotti (2010) e Anjos (2011) reafirmam a tradição da
Geografia humanística, bem como Ribeiro (2006). Nesta abordagem temos o espaço vivido, geograficidade e o lugar como conceitos centrais de análise. Pelo fato de que esta corrente de pensamento geográfico desenvolveu tradicionalmente diversos estudos de obras literárias, nota-se um foco no tratamento dos significados construídos através das letras das canções.

Visualizam-se o eixo das geografias social e cultural com uma pluralidade de abordagens. $\mathrm{Na}$ presente interpretação chama-se Geografia Cultu-

\begin{tabular}{|c|c|c|c|c|c|c|}
\hline \multicolumn{7}{|c|}{ Trabalhos sobre Geografia e música em PPG's de Geografia } \\
\hline Ano & Autor & Titulo & Tipo* & Local & Orientador & Palavras-chave \\
\hline 1991 & $\begin{array}{l}\text { João Baptista Ferreira } \\
\text { de Mello }\end{array}$ & $\begin{array}{l}\text { O Rio de Janeiro dos compositores de música popular brasileira } \\
\text { 1928/1991:uma introdução à Geografia Humanistica }\end{array}$ & M & UFRJ & Roberto Lobato Correa & $\begin{array}{l}\text { Geografia; humanismo; } \\
\text { espaço; lugar; música } \\
\text { popular }\end{array}$ \\
\hline 2000 & $\begin{array}{l}\text { João Baptista Ferreira } \\
\text { de Mello }\end{array}$ & $\begin{array}{l}\text { Dos espaços da escuridão aos lugares de extrema luminosidade - o } \\
\text { universo da estrela Marlene como palco e documento para a } \\
\text { construçäao de conceitos geográficos }\end{array}$ & D & UFRJ & Roberto Lobato Correa & $\begin{array}{l}\text { Geografia Humanistica; } \\
\text { Lugar, espaço; individuo. }\end{array}$ \\
\hline 2001 & Gisele Santos Laitano & $\begin{array}{l}\text { Os Territírios, os Lugares e a Subjetividade: construindo a } \\
\text { geograficidade pela escrita no Movimento Hip Hop, no Bairro } \\
\text { Restinga, em Porto Alegre, RS }\end{array}$ & M & UFRGS & Nelson Rego & $\begin{array}{l}\text { Território, lugar, } \\
\text { subjetividade, hermenêutica }\end{array}$ \\
\hline 2001 & $\begin{array}{l}\text { Glauco Vieira } \\
\text { Fernandes }\end{array}$ & A territorialidade sertaneja no cancioneiro de Luiz Gonzaga & M & UECE & Luiz Cruz Lima & \begin{tabular}{l|} 
Luiz Gonzaga; \\
territorialidade
\end{tabular} \\
\hline 2001 & $\begin{array}{l}\text { Nelson da Nóbrega } \\
\text { Fernandes }\end{array}$ & $\begin{array}{l}\text { Festa, cultura popular e identidade nacional: as escolas de samba } \\
\text { do Rio de Janeiro }\end{array}$ & D & UFRJ & Iná Elias de Castro & $\begin{array}{l}\text { Geografia Cultural; cultura } \\
\text { popular, festa popular }\end{array}$ \\
\hline 2002 & Luiz Felipe Ferreira & $\begin{array}{l}\text { O lugar do carnaval: espaço e poder na festa carnavalesca do Rio } \\
\text { de Janeiro. Paris e Nice (1850-1930) }\end{array}$ & M & UFRJ & Scott William Hoefle & $\begin{array}{l}\text { Carnaval; festa; lugar, Rio } \\
\text { de Janeiro; Nice; Paris }\end{array}$ \\
\hline 2002 & $\begin{array}{l}\text { Nilo Américo } \\
\text { Rodrigues Lima de } \\
\text { Almeida }\end{array}$ & $\begin{array}{l}\text { Do território dos Sentidos ocupados à sintonia com o entorno - um } \\
\text { canto para a música na Geografia }\end{array}$ & M & USP & $\begin{array}{l}\text { Maria Adélia Aparecida } \\
\text { de Souza }\end{array}$ & $\begin{array}{l}\text { música popular, geografia, } \\
\text { cultura, uso do territónio }\end{array}$ \\
\hline 2005 & Denise Prates Xavier & $\begin{array}{l}\text { Repensando a periferia no período popular da história: o uso do } \\
\text { território pelo movimento Hip Hop }\end{array}$ & & UNESP-RC & Samira Peduti Kahil & $\begin{array}{l}\text { Globalização; periferia; } \\
\text { movimmento hip hop }\end{array}$ \\
\hline 2005 & $\begin{array}{l}\text { Glauco Bruce } \\
\text { Rodrigues }\end{array}$ & $\begin{array}{l}\text { Geografias Insurgentes: um Olhar libertário sobre a Produção do } \\
\text { Espaço Urbano Através das Práticas do Movimento Hip Hop }\end{array}$ & M & UFRJ & $\begin{array}{l}\text { Marcelo Lopes de } \\
\text { Souza }\end{array}$ & $\begin{array}{l}\text { Espaço geográfico; } \\
\text { movimento hip hop; olhar } \\
\text { geográfico; }\end{array}$ \\
\hline 2005 & Marcelo Pereira Matos & $\begin{array}{l}\text { O Rio de Janeiro das Escolas de Samba: Lugar, Identidade e } \\
\text { Imagem Urbana }\end{array}$ & M & UNESP-RC & $\begin{array}{l}\text { Solange Terezinha de } \\
\text { Lima Guimarães }\end{array}$ & $\begin{array}{l}\text { Carnaval, lugar, identidade, } \\
\text { imagem }\end{array}$ \\
\hline 2006 & $\begin{array}{l}\text { Cláudia Regina Vial } \\
\text { Ribeiro }\end{array}$ & $\begin{array}{l}\text { Espaço-vivo: as variáveis de um espaço-vivo investigadas na cidade } \\
\text { de Diamantina, do ponto de vista dos músicos }\end{array}$ & D & PUCMG & $\begin{array}{l}\text { Oswaldo Bueno } \\
\text { Amorim Filho }\end{array}$ & $\begin{array}{l}\begin{array}{l}\text { Geografia Humanistica; } \\
\text { arquitetura; Urbanismo }\end{array} \\
\end{array}$ \\
\hline 2006 & $\begin{array}{l}\text { Denilson Araüjo de } \\
\text { Oliveira }\end{array}$ & $\begin{array}{l}\text { Territorialidades no Mundo Globalizado: outras leituras de Cidade a } \\
\text { partir da cultura Hip Hop }\end{array}$ & M & UFF & Jorge Luiz Barbosa & $\begin{array}{l}\text { Hip Hop, Estratégias } \\
\text { Teritorias }\end{array}$ \\
\hline 2006 & Paola Verri de Santana & Maracatu: a centralidade da periferia. & M & USP & $\begin{array}{l}\text { Ana Fani Alessandri } \\
\text { Carlos }\end{array}$ & \begin{tabular}{|l} 
Periferia, centralidade, \\
Maracatu, recife- \\
Pernambuco-Brasil
\end{tabular} \\
\hline 2007 & $\begin{array}{l}\text { Ana Carolina Viana } \\
\text { Guimarães }\end{array}$ & $\begin{array}{l}\text { Alegorias, requebros, memória e construção dos lugares do } \\
\text { carnaval carioca }\end{array}$ & M & UERJ & $\begin{array}{l}\text { João Baptista Ferreira } \\
\text { de Mello }\end{array}$ & $\begin{array}{l}\text { Geografia humanistica; } \\
\text { Canaval; Rio de Janeiro; } \\
\text { Espaço }\end{array}$ \\
\hline 2007 & Nanci Frangiotti & O espaço do carnaval na periferia da cidade de São Paulo & M & USP & $\begin{array}{l}\text { Glória da Anunciação } \\
\text { Alves }\end{array}$ & $\begin{array}{l}\text { Carnaval, uso do espaço, } \\
\text { periferia, urbanizaçẫo }\end{array}$ \\
\hline 2008 & $\begin{array}{l}\text { Alexandro Francisco } \\
\text { Camargo }\end{array}$ & $\begin{array}{l}\text { Festas Rave: uma abordagem da Geografia Psicolõgica na } \\
\text { identificação de territórios autônomos }\end{array}$ & M & UFMT & Marinete Covezzi & $\begin{array}{l}\text { Geografia Psicológica, festa } \\
\text { rave, Zona autonoma } \\
\text { temporária }\end{array}$ \\
\hline 2008 & Vanir de Lima Belo & $\begin{array}{l}\text { O enredo do Carnaval nos enredos da cidade dinâmica territorial das } \\
\text { escolas de samba em São Paulo }\end{array}$ & M & USP & Maria Mónica Arroyo & $\begin{array}{l}\text { Carnaval. Cidade, Cultura, } \\
\text { Escolas de Samba, São } \\
\text { Paulo }\end{array}$ \\
\hline 2009 & Alessandro Dozena & As territorialidades do samba na cidade de São Paulo & D & USP & $\begin{array}{l}\text { Francisco Capuano } \\
\text { Scarlato }\end{array}$ & $\begin{array}{l}\text { territorialidades, samba, } \\
\text { história, São Paulo, } \\
\text { urbanização. }\end{array}$ \\
\hline 2009 & $\begin{array}{l}\text { Daniel de Castro } \\
\text { Fernandes Coelho }\end{array}$ & Heitor Villa-Lobos: a espacialidade na alma brasileira & M & UFRJ & Roberto Lobato Correa & $\begin{array}{l}\text { Geografiá Cultural; Villa- } \\
\text { Lobos; interpretação; } \\
\text { música }\end{array}$ \\
\hline 2009 & Marcos Alberto Torres & $\begin{array}{l}\text { A paisagem sonora da llha dos Valadares: percepção e memória na } \\
\text { construção do espaço }\end{array}$ & M & UFPR & Salete Kozel Teixeira & $\begin{array}{l}\text { Paisagem sonora; } \\
\text { fandango; percepção; } \\
\text { memória }\end{array}$ \\
\hline 2009 & $\begin{array}{l}\text { Marcos Antonio } \\
\text { Correia }\end{array}$ & $\begin{array}{l}\text { Representaçãa - a música nas aulas de geografia: emoção e razão } \\
\text { nas representaçôes geográficas }\end{array}$ & M & UFPR & Salete Kozel Teixeira & $\begin{array}{l}\text { Geografia Escolar, Música; } \\
\text { Mapas Mentais }\end{array}$ \\
\hline 2009 & $\begin{array}{l}\text { Michel Rosadas dos } \\
\text { Santos }\end{array}$ & $\begin{array}{l}\text { Nascentes e Tributários de um Rio Musical - Salve Estácio, Cidade } \\
\text { Nova e a Praça Onze dos Bambas! A Vila de Noel "... só quer } \\
\text { Mostrar que faz Samba Também... }\end{array}$ & M & UERJ & $\begin{array}{l}\text { João Baptista Ferreira } \\
\text { de Mello }\end{array}$ & $\begin{array}{l}\text { Samba; Rio de Janeiro; } \\
\text { Geografia Humanistica }\end{array}$ \\
\hline 2010 & $\begin{array}{l}\text { Alexandre Moura } \\
\text { Pizotti }\end{array}$ & $\begin{array}{l}\text { Mangueira: um Simbólico Lugar Forjado no Ritmo do Sambo e no } \\
\text { Passo de seus Desfilantes }\end{array}$ & M & UERJ & $\begin{array}{l}\text { João Baptista Ferreira } \\
\text { de Mello }\end{array}$ & $\begin{array}{l}\text { Geografia humanistica, } \\
\text { Cultura popular, Mangueira; } \\
\text { Rio de Janeiro }\end{array}$ \\
\hline 2010 & $\begin{array}{l}\text { Clarissa Valadares } \\
\text { Xavier. }\end{array}$ & $\begin{array}{l}\text { Festas e micaretas - a mistura elètrica da alegria: pelas vias, veias } \\
\text { culturais e modelagem turística }\end{array}$ & M & UFG & $\begin{array}{l}\text { Carlos Eduardo Santos } \\
\text { Maia }\end{array}$ & $\begin{array}{l}\text { Carnaval, festas, espaço, } \\
\text { cultura, micareta }\end{array}$ \\
\hline 2010 & Lucas Manassi Panitz & $\begin{array}{l}\text { Por uma geografia da música: o espaço geográfico da música } \\
\text { popular platina }\end{array}$ & M & UFRGS & Álvaro Luiz Heidrich & $\begin{array}{l}\text { Espaço platino; } \\
\text { templadismo; estética do } \\
\text { frio; representaçôes do } \\
\text { espaço }\end{array}$ \\
\hline 2011 & Bruno Picchi & $\begin{array}{l}\text { De homens e caranguejos ao Caranguejos com Cérebro: a região } \\
\text { cultural do Movimento Manguebit e o Recife contemporâneo }\end{array}$ & M & UNESP-RC & \begin{tabular}{l|} 
Paulo Roberto Teixeira \\
de Godoy
\end{tabular} & $\begin{array}{l}\text { Movimento Manguebit; } \\
\text { Região; Cultura; Cidade; } \\
\text { Música; Regiäo }\end{array}$ \\
\hline 2011 & $\begin{array}{l}\text { Fernando Lucci } \\
\text { Resende de Souza }\end{array}$ & $\begin{array}{l}\text { Composição urbana, ritmos e melodias de uma geografia de vida, } \\
\text { Villa-Lobos o moderno compositor carioca: Na trilha dos Choros }\end{array}$ & M & UFF & $\begin{array}{l}\text { Marcio Piñon de } \\
\text { Oliveira }\end{array}$ & $\begin{array}{l}\text { Ciência geográfica; } \\
\text { geobiografia; Heitor Villa- } \\
\text { Lobos:Choros }\end{array}$ \\
\hline 2011 & Juliana Cunha Costa & $\begin{array}{l}\text { Segregação espacial e música eletrônica: a cena cultural } \\
\text { soteropolitana }\end{array}$ & M & UFBA & $\begin{array}{l}\text { Maria A } \\
\text { Silva }\end{array}$ & $\begin{array}{l}\text { Música eletrônica; } \\
\text { Carnaval; Raves; } \\
\text { Segregação; Espaço } \\
\text { Urbano }\end{array}$ \\
\hline 2011 & $\begin{array}{l}\text { Melissa Souza dos } \\
\text { Anjos }\end{array}$ & Lugares e personagens do universo Buarqueano & M & UERJ & $\begin{array}{l}\text { João Baptista Ferreira } \\
\text { de Mello }\end{array}$ & $\begin{array}{l}\text { Geografia Humanistica; } \\
\text { Lugar, Música; } \\
\text { Subjetividade }\end{array}$ \\
\hline 2012 & $\begin{array}{l}\text { Carlos Geovani Ramos } \\
\text { Machado** }\end{array}$ & O Ensino de geografia e o Hip Hop & M & UFRGS & $\begin{array}{l}\text { Antônio Carlos } \\
\text { Castrogiovanni }\end{array}$ & $\begin{array}{l}\text { Ensino de Geografia; hip } \\
\text { hop; lugar, território; } \\
\text { complexidade }\end{array}$ \\
\hline & & & & & & \\
\hline
\end{tabular}

Quadro 1. Trabalhos defendidos nos PPG's em Geografia (Elaboração: Lucas Panitz). 
ral e Social um grande filão de interesses e tratamentos teóricos mais ou menos aproximados, que visualizam a música como um elemento que envolve produção do espaço, uso do território, criação de identidades, territorialidades, regionalidades e representações do espaço. Nesta, vemos interpretações ora mais ligadas às geografias críticas, ora em direção à geografia cultural renovada de base francesa e anglo-saxã, ou ainda uma combinação de ambas. Os interesses particulares são heterogêneos, porém concentram-se sobretudo no estudo do samba e do carnaval (FERNANDES, N., 2001; FERREIRA, 2002; MATOS, 2005; FRANGIOTTI, 2007; BELO, 2008; DOZENA, 2009, XAVIER, C., 2010), do hip hop (LAITANO, 2001; XAVIER, D., 2005; OLIVEIRA, 2006; RODRIGUES, 2005) e música erudita (CASTRO, 2009a; SOUZA, 2011). Além disso, se visualiza trabalhos sobre a música do nordeste - forró, maracatu, movimento manguebit - (FERNANDES, G., 2001; SANTANA, 2006; PICCHI, 2011), música eletrônica (CAMARGO, 2008; COSTA, 2011), além da música popular em um contexto mais geral, como a cena musical paulistana em Almeida (2002), o fandango em Torres (2009), e a música popular transfronteiriça entre Brasil, Argentina e Uruguai, em Panitz (2010)

Uma terceira abordagem, em Correia (2009) e Machado (2012), indica a relação entre a música e o ensino de Geografia. Nessa abordagem, que não exclui aproximações humanistas ou da Geografia Social e Cultural, o foco é o uso da música para construir conceitos geográficos em sala de aula.

Analisando o Gráfico 1, é possível ver que $80 \%$ dos trabalhos concentram-se em três áreas de interesse: o Samba e o Carnaval, a Música Popular Brasileira em suas diversas manifestações, e finalmente o rap e o movimento hip hop. Como assuntos ainda periféricos têm-se a música erudita, a música eletrônica e as festas rave, e a música transfronteiriça. Nota-se, também, que $75 \%$ da produção acadêmica na área provem das regiões sul e sudeste do país - esta última com dois terços da produção total dos trabalhos. É possível entender que a primazia dos trabalhos em samba, carnaval e hip hop estão atrelados a essa relação (ver Gráfico 2).

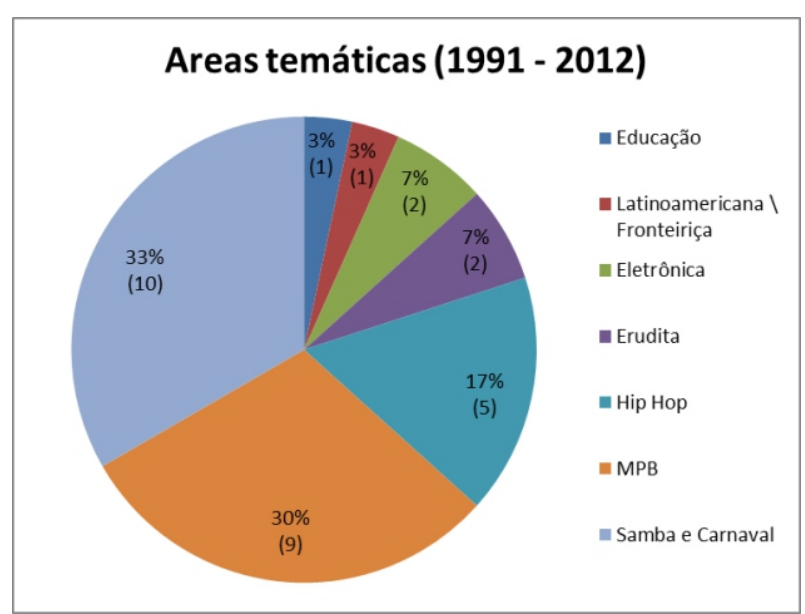

Gráfico 1. Distribuição dos trabalhos em percentual e números absolutos (Elaboração: Lucas Panitz).

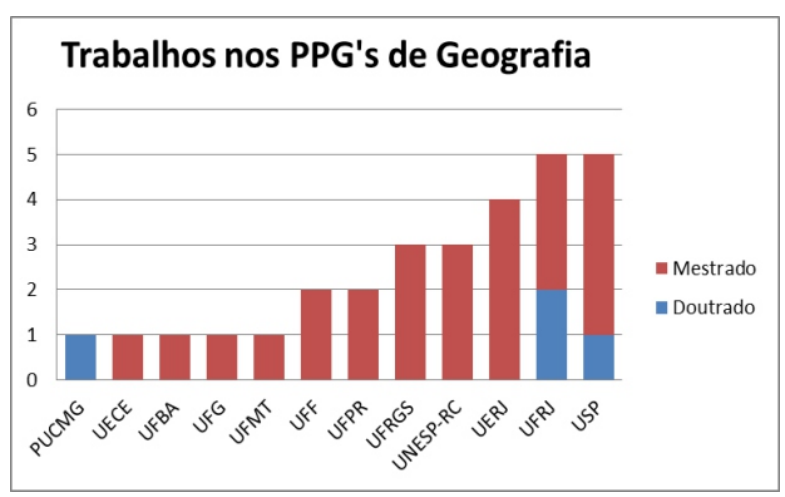

Gráfico 2. Distribuição dos trabalhos por Universidade (Elaboração: Lucas Panitz).

É possível ainda citar produções bibliográficas em periódicos, livros e coletâneas de Geografia. Encontramos em Mesquita (1994, 1997) algumas das primeiras publicações no Brasil, advogando por uma Geografia da música social nos territórios fronteiriços do Prata. Campos (2006), por sua vez, oferece o potencial da música popular para a análise geográfica no ensino fundamental e médio, no que toca as questões do ambiente e da cultura nordestina do semiárido. Ainda voltados ao ensino, Correia e Kozel (2009) voltam-se para uma geografia fenomenológica e das representações sociais, para discutir as ressignificações dos conteúdos geográficos por meio da música. Evangelista trata em recente livro as distintas "ambiências espaciais" do samba, da bossa-nova, do rock e do funk. Cardoso (2009), por sua vez, realizou uma análise da geografia urbana de São Paulo através das canções do compositor Itamar Assumpção. Guimarães propõe reflexões teóricas sobre a relação entre a escala 
musical e a escala geográfica com base em considerações musicológicas do pesquisador e compositor José Miguel Wisnik e as concepções de escala geográfica do geógrafo Roger Brunet. Em Castro (2009b), por fim, reconhecemos um esforço em remontar o interesse geográfico pela música, sobretudo a partir da Geografia anglo-saxã.

\section{Geografia e música no Brasil: algumas conside- rações}

Vimos que nos países anglo-saxões, por exemplo, há dois momentos distintos: a abordagem difusionista da escola saueriana e a renovação crítica, inspirada nos estudos culturais. Na França mantém-se um ponto de vista territorial: a música na construção e na afirmação da identidade territorial, a produção de políticas territoriais voltas para a música, as territorializações efêmeras dos festivais, entre outros. No Brasil, contudo, nota-se uma diversidade de abordagens, interesses e tratamentos metodológicos. Estas têm valorizado significativamente nos últimos vinte anos uma parte considerável da diversidade musical do país e suas relações com as identidades regionais e nacionais, a construção de territorialidades e de discursos geográficos, e as transformações do espaço urbano. Para tanto se visualizam ao menos três abordagens (ainda que esta enumeração seja apenas expositiva, pois na prática há nuances entre as mesmas). Em um primeiro momento temos abordagens calcadas nas filosofias do significado (fenomenologia, hermenêutica, existencialismo e outros) através da Geografia Humanística - esta utilizou largamente a letra da canção como fonte de suas pesquisas. Também, abordagens em Geografia Social e Cultural as quais mantém a tradição crítica da Geografia brasileira nas últimas décadas, utilizando referenciais teóricos da sociologia, antropologia, história cultural e estudos culturais - nestas utiliza-se não só as letras das canções, como também a perspectiva do som, as espacialidades da atividade musical e os discursos dos atores produtores da música. Por fim, as abordagens voltadas ao ensino em Geografia, produzindo compreensões para a construção de conceitos geográficos.

É importante ressaltar o reconhecimento por parte da Geografia brasileira, considerando o papel da música (seja ela popular, eletrônica ou erudita) para a produção do espaço geográfico em suas mais diversas formas. Nesse sentido, a diversidade de interesses apresentada e a indiscutível riqueza musical do país, fazem deste campo de estudo um lugar fecundo para explorar o espaço geográfico em suas mais diversas abordagens e já tem oferecido, sem dúvidas, novos olhares para as relações entre espaço e cultura.

\section{Referências}

ANDERSON, Ben; MORTON, Frances; REVILL, George. Practices of music and sound. Social \& Cultural Geography, v. 6, n. 5, 2005, 639-644. <http: //tinyurl.com/436e5nu>. [20 de julho de 2011].

ANJOS, Melissa Souza. Lugares e personagens do universo Buarqueano. Dissertação de mestrado dirigida por João Baptista Ferreira de Mello. Rio de Janeiro: Universidade Estadual do Rio de Janeiro, 2011.

BELO, Vanir de Lima. 0 enrodo do Carnaval nos enredos da cidade : dinâmica territorial das escolas de samba em São Paulo. Dissertação de mestrado dirigida por María Mónica Arroyo. São Paulo : Universidade de São Paulo, 2008.

CALENGE, Pierre. Les territoires de l'innovation: les réseaux de l'industrie de la musique en recomposition Territories of innovation: the transformation of the music industry networks. Géographie, Economie, Société, $\mathrm{C}$ a c h a n, v. 4, 2002, 37 - 56 . $<$ http://tinyurl.com/3k4e8aq>. [20 de julho de 2011].

CAMARGO, A. Festas . Rave: uma abordagem da Geografia Psicológica na identificação de Territórios Autônomos. Dissertação de Mestrado dirigida por Marinete Covezzi. Cuiabá: Universidade Federal do Mato Grosso, 2008. <http:// tinyurl.com/3kr5tu3>. [20 de julho de 2011]

CAMPOS, Rui Ribeiro de. A Geografia da Semi-Aridez Nordestina e a MPB. Revista Sociedade \& Natureza, Uberlândia, 18 (35), 2006, 169-209.

CARDOSO, Eduardo.Schiavone. A metrópole na linha do baixo: Itamar Assumpção e a geografia da cidade de São Paulo. Espaço e Cultura 25, 2009, 31-40.

CASTRO, Daniel. Geografia e música: a dupla face de uma relação. Espaço e Cultura 26, p.7-18, Jul/Dez, 2009b.

CASTRO, Daniel. Heitor Villa-Lobos: a espacialidade na alma brasileira. Dissertação de mestrado dirigida por Roberto Lobato Correa. Rio de Janeiro: Universidade Federal do Rio de Janeiro, 2009a.

CONNELL, John. GIBSON, Chris. World music: deterritorializing place and identity. Progress in Human Geography 28, 2004, 342. <http:// tinyurl.com/428jj9o>. [20 de julho de 2011].

CORREIA, Marcos Alberto. KOZEL, Salete. Representação e Ensino: Ressignificação de conteúdos geográficos por meio da música. Disponível e m: <http://tiny.cc/srkid>. [20 de novembro de 2009]

CORREIA, Marcos Alberto. Representação e ensino, a música nas aulas de geografia: razão e emoção nas representações geográficas. Dissertação de Mestrado dirigida por Salete Kozel. Curitiba: Universidade Federal do Paraná, 2009. 116p. <http://tinyurl.com/3e8ltoz>. [20 de julho de 2011].

COSTA, Juliana Cunha. Segregação espacial e música eletrônica: a cena cultural de Salvador e Camaçar. Dissertação de Mestrado dirigida por Maria Auxiliadora. Salvador: Universidade Federal da Bahia, 2010.89p. 
DOZENA, Alexandro. As Territorialidades do Samba na Cidade de São Paulo. Tese de Doutorado dirigida por Francisco Capuano Scarlato. São Paulo: Universidade de São Paulo, 2009. 266p. <http://tinyurl.com/3jyswyy>. [20 de julho de 2011].

DUNCAN, James. The superorganic in American cultural geography. Annals of the Association of American Geographers 1980, 181-198.

EVANGELISTA, Hélio Araújo. Rio de Janeiro e a música. Uma reflexão sobre a decadência, a carioca e a da própria música. Rio de Janeiro: Armazém Digital, 2005.

FERNANDES, Glauco Vieira. "Reterritorialização" Da Cultura Sertaneja Em Luiz Gonzaga. Cadernos de Cultura e Ciência, v. 3, n. 1, 2009. <http:// tinyurl.com/3srvs9n>. [20 de julho de 2011].

FERNANDES, Nelson da Nóbrega. Escolas de samba: sujeitos celebrantes e objetos celebrados. Rio de Janeiro: S e cretaria das Culturas, 2001 . http://tinyurl.com/3fzh4az>. [20 de julho de 2011].

FERREIRA, Luiz Felipe. O lugar do carnaval: espaço e poder na festa carnavalesca do Rio de Janeiro, Paris e Nice (1850-1930). Tese de doutorado dirigida por Scott William Hoefle. Rio de Janeiro: Universidade Federal do Rio de Janeiro, 2002.

FINN, John. Contesting culture: a case study of commodification in Cuban music. GeoJournal, v. 74, n. 3, 2009,191-200. <http://tinyurl.com/3gxuqph>. [20 de julho de 2011].

FLORIDA, Richard.JACKSON, Scott. Sonic city: the evolving economic geography of the music industry. Journal of Planning Education and Research 29, 2010, 310-321. <http:// tinyurl.com/4xkqyzg>. [20 de julho de 2011].

FRANGIOTTI, Nanci. O espaço do carnaval na periferia da cidade de São Paulo. Dissertação de mestrado dirigida por Glória da Anunciação Alves. São Paulo : Universidade de São Paulo, 2007.

GIBSON, Chris. "We sing our home, We dance our land": indigenous self-determination and contemporary geopolitics in Australian popular music. Environment and P l a n n i n g D 16, $1998,163-184$. $<$ http://tinyurl.com/3mumxfp>. [20 de julho de 2011].

GIBSON, Chris. Place and Music: performing'the region'on the New South Wales Far North Coast. Transfor$\mathrm{ming} \mathrm{Cultures}$ e J o u r n a 4,2009 . <http://tinyurl.com/3dou7eq>. [20 de julho de 2011].

GIRONCOURT, Georges. La géographie musicale. La Géographie XLVIII, 1927, 292-302. <http: //tinyurl.com/3e8shrk>. [20 de julho de 2011].

GIRONCOURT, Georges. Recherces de Géographie musicale dans le Sud Tunisien. La Géographie, v. LXXL, n. 6 , 1939, 65-74.

GORÉ, Olivier. L'inscription territoriale de la musique traditionnelle en Bretagne. Thèse de doctorat dirigé par Jean Pihan. Rennes: Université de Rennes, 2004. 421p. <http:// tinyurl.com/3cl4fux>. [20 de julho de 2011].

GUIMARAES, Ana Carolina Viana. Alegorias, requebros, memória e construção dos lugares do carnaval carioca. Dissertação de mestrado dirigida por João Baptista Ferreira de Mello. Rio de Janeiro: Universidade Estadual do Rio de Janeiro, 2007.
GUIMARAES, Raul Borges. Escala Geográfica e Partitura Musical: Considerações Acerca do Sistema Modal e Tonal. In: CORRÊA, R. L.; ROSENDAHL, Z. Espaço e cultura: Pluralidade tematica. EdUERJ, 2008.

GUIU, Claire (dir.). Géographies et musiques : quelles perspectives? Géographie et Cultures 59, 2006.

HOGAN, Ellen. 'Enigmatic territories': geographies of popular music. Critical Public Geographies Working Paper, Department of Applied Social Studies, University College Cork, 2007. <http://tinyurl.com/3dsoysk>. [20 de julho de 2011].

HUDSON, Ray. Making music work? Alternative regeneration strategies in a deindustrialized locality: the case of Derwentside. Transactions of the Institute of British Geographers, v. 20, n. 4, 1995, .460-473. <http://tinyurl.com/42j4m4u>. [20 de julho de 2011].

JAZEEL, Tariq. The world is sound? Geography, musicology and British-Asian soundscapes. Area, v. 37, n. 3, 2005, 233-241. <http:// tinyurl.com/43qfuhm>. [20 de julho de 2011].

KEARNEY, Daithí. Crossing the River: Exploring the Geography of Irish Traditional Music. Journal of the Society for Musicology in Ireland 3, 2008, 127-139. <http://tinyurl.com/3g45y8g>. [20 de julho de 2011].

KINGSBURY, Aaron. Music in the Fields: Constructing Narratives of the Late 19 th Century Hawaiian Plantation Cultural Landscape. Yearbook of the Association of Pacific Coast Geographers 70, 2008, 45-58. < http://tinyurl.com/3uhmdww>. [20 de julho de 2011].

KONG, Lily. Making "music at the margins"? A social and cultural analysis of Xinyao in Singapore. Asian Studies $\mathrm{R}$ e vi e w $19,1996,99-124$ < http://tinyurl.com/3r35bw4>. [20 de julho de 2011].

KONG, Lily. Popular music in a transnational world: the construction of local identities in Singapore. Asia Pacific $\mathrm{V}$ i e w p o i n t $38,1997,19-36$. http://tinyurl.com/4x4s2b4>. [20 de julho de 2011].

KONG, Lily. Popular music in geographical analyses. Progress in Human Geography 19, 1995, 183-183. $<$ h ttp: / / profile.nus.edu.sg/fas s / geokongl/pihg19.pdf>. [20 de julho de 2011].

KRUSE, Robert. The Geography of the Beatles: Approaching Concepts of Human Geography. Journal of Geog raphy, v. $103, \mathrm{n} .1, \mathrm{p} .2-7,2004$. <http://tinyurl.com/3pbn34g>. [20 de julho de 2011].

LAMANTIA, Frédéric. Les effets" territorialisants" des sons, reflets de la société en ses lieux et de ses états d'âme. Gé oc arrefour 78/2, 2003,173-175. <http://tinyurl.com/43uweek>. [20 de julho de 2011].

LECHAUME, Aline. Chanter le pays : sur les chemins de la chanson québécoise contemporaine. Géographie et Cultures, n.21, 1997, 45-58.

LEROUX, Xavier. Pour une Géographie de la musique traditionelle dans le nord de la France. Bulletin de la Société géographique de Liège $49,2007,59-65$. < http://tinyurl.com/3covpca>. [20 de julho de 2011].

LÉVY, Jacques. Espace, contrepoints-Des géographies musicales. Espaces-Les cahiers de l'IRCAM/Recherche et musique, 1994, 145-166.

LÉVY, Jacques. Le tournant géographique. Paris: Belin, 
1999.

LEYSHON, Andrew. MATLESS, David. REVILL, George. The place of music. New York: Guilford Press, 1998.

LIMA, Nilo. Dos territórios dos sentidos ocupados à sintonia com o entorno - um canto para a música na geografia. Dissertação de Mestrado em Geografia dirigida por Maria Adélia Aparecida de Souza. São Paulo: Universidade de São Paulo, 2002.

MACHADO, Carlos Geovani Ramos. O Ensino de Geografia e o Hip Hop. Dissertação de mestrado dirigida por Antônio Carlos Castrogiovanni. Porto Alegre : Universidade Federal do Rio Grande do Sul, 2012.

MELLO, João Baptista Ferreira. Dos Espaços da Escuridão aos Lugares de Extrema Luminosidade - O Universo da Estrela Marlene como Palco e Documento para a Construção de Conceitos Geográficos. Tese de doutorado dirigida por Roberto Lobato Correa. Rio de Janeiro: Universidade Federal do Rio de Janeiro, 2000.

MELLO, João Baptista Ferreira. O Rio de Janeiro dos Compositores da música popular brasileira -1928/1991 uma introdução à geografia humanística. Dissertação de Mestrado dirigida por Roberto Lobato Correa. Rio de Janeiro: Universidade Federal do Rio de Janeiro, 1991.

MERRIAM, Alan. Antropologia della musica. Palermo: Sellerio, 1983.

MESQUITA, Zilá. A geografia social na música do Prata. Espaço e Cultura. 3, 1997, 33-41.

MESQUITA, Zilá. A pauta musical da fronteira: um convite à Geografia cultural. In: CASTELLO. I.R. et al. Práticas de Integração nas fronteiras: temas para o Mercosul. Porto Alegre: Ed. Universidade/UFRGS, 1994. p.176182.

NASH, Peter. CARNEY, George. The seven themes of music geography. Canadian Geographer 40, 1996, 69-74. <http://tinyurl.com/3qbz5ac>. [20 dejulho de 2011].

OLIVEIRA, Denilson Araújo. Territorialidades no Mundo Globalizado: outras leituras da cidade a partir da cultura hip hop. Dissertação de Mestrado dirigida por Jorge Luiz Barbosa. Niterói: Universidade Federal Fluminense, 2006.169p.

PANITZ, Lucas Manassi. Por uma geografia da música: as representações do espaço geográfico na música popular platina. Dissertação de Mestrado dirigida por Álvaro Luiz Heidrich. Porto Alegre: Universidade Federal do Rio G r a n d e d o S u l, 20100.200 . <http://tinyurl.com/4ycfdmz>. [20 de julho de 2011].

PANITZ, Lucas Manassi. Geografia e música: uma introdução ao tema. Biblio 3W. Revista Bibliográfica de Geografía y Ciencias Sociales. [En línea]. Barcelona: Universidad de Barcelona, 30 de mayo de 2012, Vol. XVII, $\mathrm{n}^{-}$ 978. <http:// www.ub.es/geocrit/b3w-978.htm>. [ISSN 1138-9796].

PICCHI, Bruno. De homens e caranguejos ao Caranguejos com Cérebro: a região cultural do Movimento Manguebit e o Recife contemporâneo. Dissertação de mestrado dirigida por Paulo Roberto Teixeira de Godoy. Rio Claro : Universidade Estadual Paulista, 2011.

PIZOTTI, Alexandre Moura. Mangueira: um Simbólico Lugar Forjado no Ritmo do Sambo e no Passo de seus Desfilantes. Dissertação de mestrado dirigida por João
Baptista Ferreira de Mello. Rio de Janeiro: Universidade Estadual do Rio de Janeiro, 2010.

RAIBAUD, Yves (dir). Comment la musique vient aux territoires. Bourdeaux: MSHA, 2008.

REVILL, George. Music and the politics of sound: nationalism, citizenship, and auditory space. Environment and Planning D 18, n. 5, 2000,597-614. <http://tinyurl.com/3k9c45x>. [20 de julho de 2011].

REVILL, George.. Vernacular culture and the place of folk music. Social and Cultural Geography 6, n. 5, 2005. 693706. <http://tinyurl.com/3bcvhhq>. [20 de julho de 2011].

REYNOSO, Carlos. Antropología de la música: De los géneros tribales a la globalización. Volumen I: Teorías de la simplicidad. Buenos Aires: Editorial Sb, 2006.

RIBEIRO, Claudia Vial. Espaço-vivo: as variáveis de um espaço-vivo investigadas na cidade de Diamantina, do ponto de vista dos músicos. Tese de Doutorado dirigida por Oswaldo Bueno Amorim Filho. Belo Horizonte: PUCMG, 2006. 289p.

RODRIGUES, Glauco Bruce. Geografias Insurgentes: um Olhar libertário sobre a Produção do Espaço Urbano Através das Práticas do Movimento Hip Hop. Dissertação de mestrado dirigida por Marcelo Lopes de Souza. Rio de Janeiro: Universidade Estadual do Rio de Janeiro, 2005.

ROMAGNAN, Jean-Marie. La musique: un nouveau terrain pour les géographes. Géographie et cultures 36, 2000, 107-126.

ROSADAS, Michel. Nascentes e tributários de um Rio musical - salve Estácio, Cidade Nova e a Praça Onze dos bambas! E a Vila de Noel "so quer mostrar que faz samba também". Dissertação de Mestrado dirigida por João Baptista Ferreira de Mello - Instituto de Geografia, Universidade do Estado do Rio de Janeiro, Rio de Janeiro, 2009.161p.

SALDANHA, Arun. Trance and visibility at dawn: racial dynamics in Goa's rave scene. Social and Cultural Geography, v. 6, n. 5, p. $707-721,2005$. <http://tinyurl.com/3hpk7kp>. [20 de julho de 2011].

SANTANA, Paola Verri. Maracatu : a centralidade da periferia. Dissertação de mestrado dirigida por Ana Fani Alessandri Carlos. São Paulo : Universidade de São Paulo, 2006.

SANTOS, Michel Rosadas. Nascentes e Tributários de um Rio Musical - Salve Estácio, Cidade Nova e a Praça Onze dos Bambas! A Vila de Noel "...só quer Mostrar que faz Samba Também.... Dissertação de mestrado dirigida por João Baptista Ferreira de Mello. Rio de Janeiro: Universidade Estadual do Rio de Janeiro, 2009.

SOUZA, Fernando Lucci Resende. Composição urbana, ritmos e melodias de uma geografia de vida, Villa-Lobos o moderno compositor carioca: Na trilha dos Choros. Dissertação de mestrado dirigida por Marcio Piñon de Oliveira. Niterói : Universidade Federal Fluminense, 2011.

TORRES, Marcos Alberto. A paisagem sonora da Ilha dos Valadares: percepção e memória na construção do espaço. Dissertação de mestrado dirigida por Salete Kozel Teixeira. Curitiba : Universidade Federal do Paraná, 2009. 
XAVIER, Clarissa. Festas e micaretas - a mistura elètrica da alegria: pelas vias, veias culturais e modelagem turística. Dissertação de mestrado dirigido por Carlos Eduardo Santos Maia. Goiânia: Universidade Federal de Goiás, 2010.
XAVIER, Denise Prates. Repensando a periferia no período popular da história: o uso do território pelo movimento Hip Hop. Dissertação de mestrado dirigida por Samira Peduti Kahil. Rio Claro: Universidade Estadual Paulista, 2005.

\title{
Geography and music: an introduction
}

\begin{abstract}
Geography of Music, despite its absence from the canon of the discipline, has gained such an important dimension for geographers concerned with cultural studies and artistic expressions in their spatial dimension. The increased availability of digital materials and archives allowed for the recognition of this field of study in geography, being the United States, the United Kingdom, France and Brazil (given its potential for ibero-american studies) important centers of debate and scholarly production. This article offers an global outlook and focuses on the twenty years of brazilian scholarly production.
\end{abstract}

Keywords: Geography. Music. Geography of Music. Brazilian Geography.

\section{Geografía y música: una introducción al tema}

Resumen - La geografía de la música, en que pese prácticamente un siglo de existencia oficial, solo recientemente tuvo la debida atención de los geógrafos interesados en estudio de la cultura y de las manifestaciones artísticas en su dimensión espacial. La cantidad de materiales disponibles en formato digital permite un bueno reconocimiento de este campo de estudios en geografía, y apunta Estados Unidos, Inglaterra y Francia como centros de discusión avanzada, además el Brasil como potencial ámbito ibero-americano, por su considerable producción científica. En este sentido, el texto presenta un panorama global de los estudios de Geografía y música, y enfoca en los veinte años de producción de la Geografia brasileña.

Palabras-clave: Geografía. Música. Geografía de la Música. Geografía brasileña. 